\title{
Implementation of Transmitter Zigbee System based on Wireless Sensor Network of IEEE 802.15.4 Standard
}

\author{
Ekhlas K. Hamza* Russul H. Hadi** \\ *,**Department of Control and Systems Engineering / University of Technology \\ *Email: Ekhlas_kadhum@yahoo.com \\ ** Email: russulh@gmail.com
}

(Received 6 December 2016; accepted 29 March 2017)

https://doi.org/10.22153/kej.2017.03.002

\begin{abstract}
Zigbee is considered to be one of the wireless sensor networks (WSNs) designed for short-range communications applications. It follows IEEE 802.15.4 specifications that aim to design networks with lowest cost and power consuming in addition to the minimum possible data rate. In this paper, a transmitter Zigbee system is designed based on PHY layer specifications of this standard. The modulation technique applied in this design is the offset quadrature phase shift keying (OQPSK) with half sine pulse-shaping for achieving a minimum possible amount of phase transitions. In addition, the applied spreading technique is direct sequence spread spectrum (DSSS) technique, which has the effect in getting better performance for the transmitter system in multipath environments. The system is developed through the use of MATLAB/Simulink to show the performance of the proposed system in response to the appliy modulation technique.
\end{abstract}

Keywords: DSSS technique, IEEE 802.15.4, MSK, OQPSK, Zigbee.

\section{Introduction}

Short-range networks are classified into two categories. The first one is Wireless Local Area Networks (WLANs), an extension to wired-Local Area Networks (LANs), and the second is Wireless Personal Area Networks (WPANs) that are also called as Wireless Sensor Networks (WSNs). Figure 1 shows some of the properties included in those two categories [1].

Although infrared, WLAN, and Bluetooth have been extensively used as near field wireless communication technologies, they have some drawbacks, including difficulty and complexity in design and installation processes, the large amount of wasted power, short coverage area, and suitable only for small-scale networking. In contrast, WSNs have a greater role in solving the inconvenience into people's lives. They have many features unlike wired-network, such as suitable organizing of the network, less affectation by surrounding environment, low-cost and low power consumption [2]. The low rate wireless personal area networks (LR-WPANs) include two types of devices that are fully functional devices (FFD) and devices with reduced functionality (RFD). The first type could be utilized as a device, a PAN coordinator, or a coordinator, while the second could be used as a device only [3].

This paper is organized as follows. IEEE 802.15.4 and Zigbee architecture are presented in Section 2. Section 3 introduces an overview of digital modulation techniques. In section 4,the proposed model is demonstrated. Simulation and design results are depicted in section 5. Finally, section 6 includes research conclusions. 


\section{IEEE 802.15.4 and Zigbee Architecture}

IEEE 802.15.4 standard identifies specifications of both of physical layer (PHY) and medium access control (MAC) sub layer for LRWPANs. It also includes extended layers for WPANs and WLANs. Figure 2 shows the architecture of Zigbee technology as an LRWPAN [4].
Zigbee technology can be worked at three various frequency bands according to the geographical coverage area. In Europe, 868-868.6 $\mathrm{MHz}(868 \mathrm{MHz})$ is used, in Northern America $902-928 \mathrm{MHz}(915 \mathrm{MHz})$, while the $2.4 \mathrm{GHz}$ is used all over the world, and it depends on unlicensed Industrial, scientific and Medical(ISM) radio bands [5].

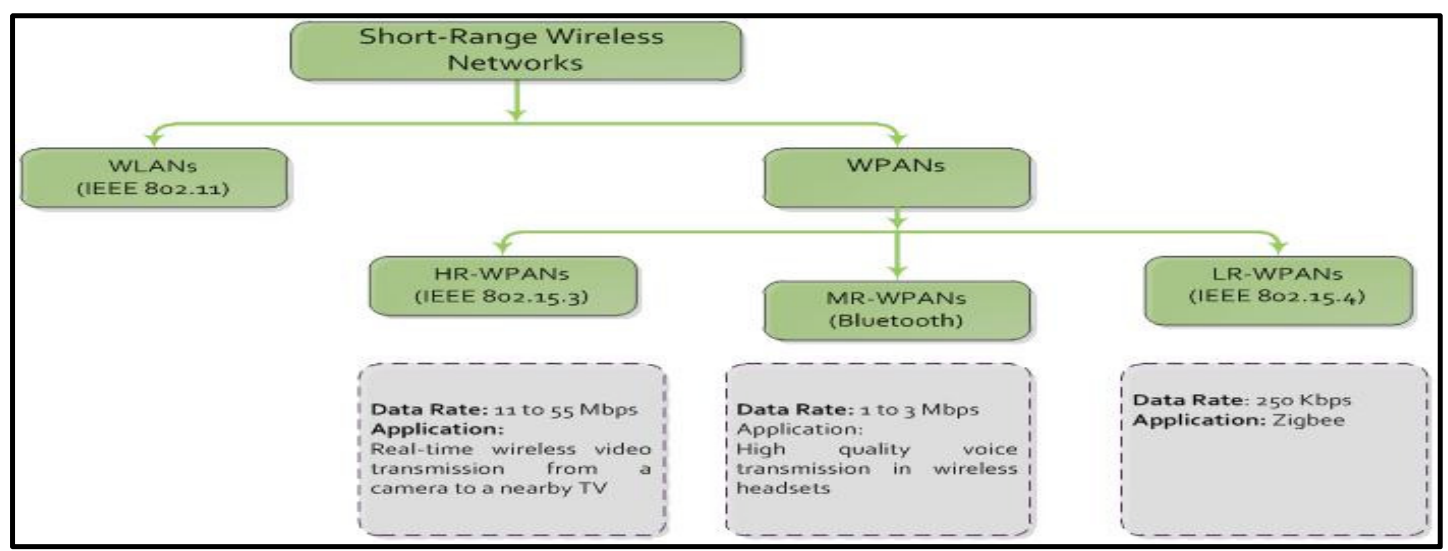

Fig. 1. Classes of Short-Range Wireless Network.

\section{Overview of Digital Modulation Techniques}

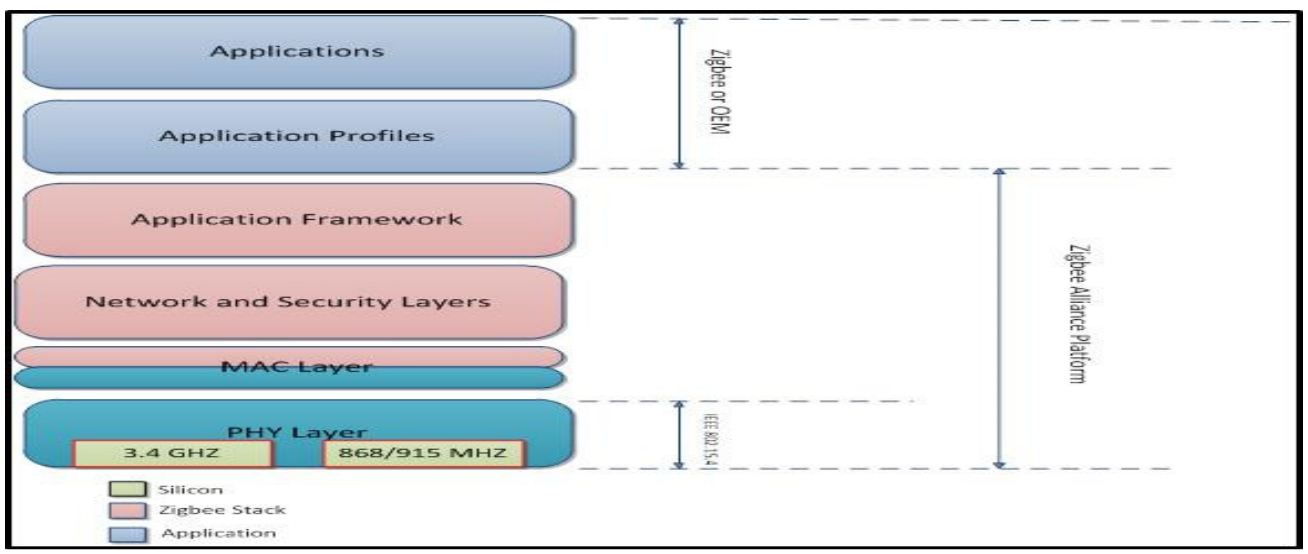

Fig. 2. Zigbee Technology Architecture.

The process that effects on a digital symbol in order to convert it into a signal suitable for transmission is called digital modulation [6]. This process involves the use of various digital modulation techniques that are different in certain parameters. Varying signal amplitude, frequency or phase could be done to obtain some of these techniques. Furthermore, hybrid techniques could be driven from hybrid variations of these parameters [7]. Figure 3 illustrates the classification of digital modulation techniques [8].
In this paper, Offset Quadrature Phase Shift Keying (OQPSK), also called Staggered QPSK (SQPSK), modulation technique is applied, which is the modified version of QPSK. The benefit behind this modification is to delay the signal bya half cycle so as to avoid possible change in signal phase. The carrier permits to send the signal for four possible orthogonal phases and as shown in figure 4 [6] [9] [10] [11]. 


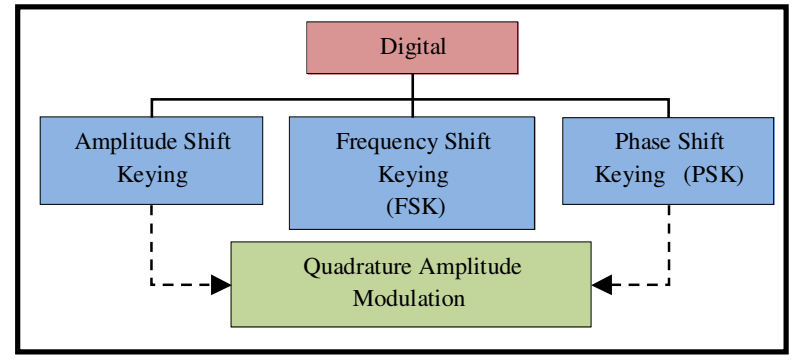

Fig. 3. Types of Digital Modulation.
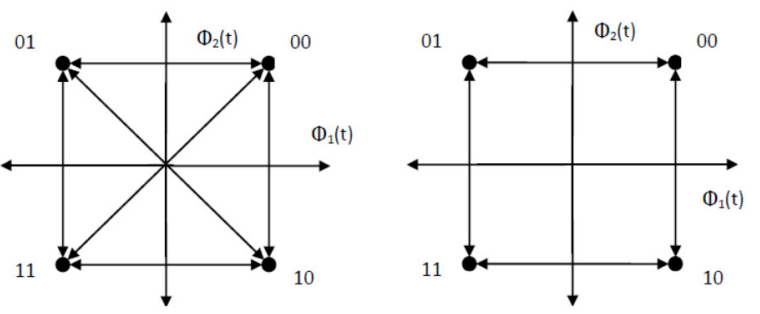

Fig. 4. Constellation Plot of QPSK and OQPSK.

In OQPSK, just like QPSK, the data stream mk $(\mathrm{t})=\mathrm{m} 0, \mathrm{~m} 1, \mathrm{~m} 2, \ldots$, is divided into two channels: In-phase and Quadrature. In-phase channel represents the even bit streams of data $\mathrm{mI}(\mathrm{t})=$ $\mathrm{m} 0, \mathrm{~m} 2, \mathrm{~m} 4$. . ., while $\mathrm{mQ}(\mathrm{t})=\mathrm{m} 1, \mathrm{~m} 3, \mathrm{~m} 5, . . .$, represents the odd bit streams for Quadrature channel that are staggered by only one bit period (half a symbol period) and this is depicted in Figure 5. OQPSK waveform is shown in Figure 6 which clarifies that the phase shift does not exceed $\pm 90^{\circ}$ hence; the waveform envelope will be limited as it will not go to zero as it does with QPSK [12]. In addition, symbol interference effect caused by limited transmitted band and non-linear behavior of the used amplifier could be reduced[6]. For further enhancements, it is possible to reduce phase changes to produce a smoother signal that could behave as completely continuous. One type of continuous phase frequency shift keying (CPFSK) is a minimumshift keying (MSK) modulation technique. MSK could be produced from OQPSK by shaping the pulses with half sine wave, or can be driven as a special case of CPFSK. As compared with QPSK or OQPSK, the MSK modulation technique has some features, including constant envelop of transmitted signal and continuous phase (no sudden phase changes) [6][13].

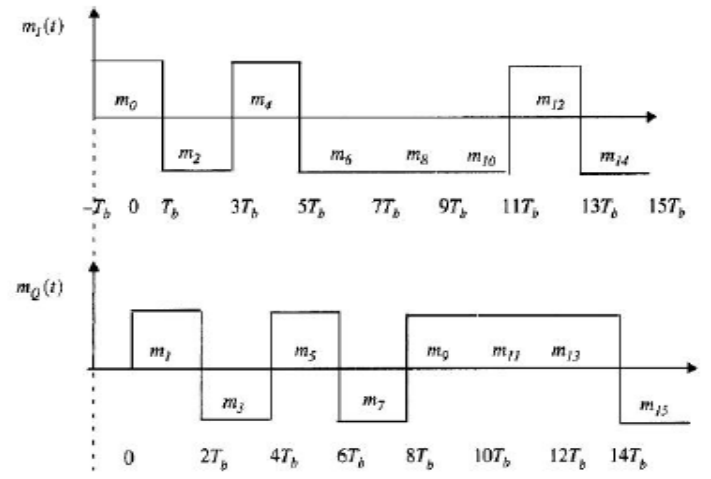

Fig. 5. Staggering of the In-phase and Quadrature modulated bit streams in OQPSK.

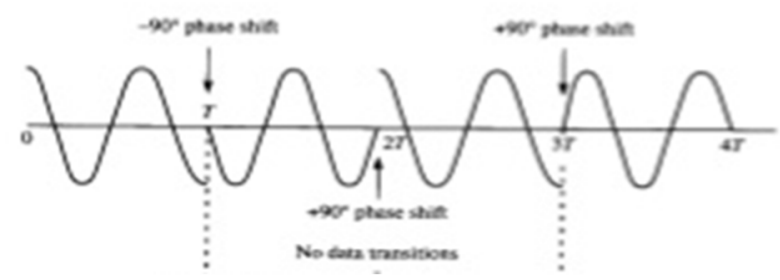

Fig. 6.OQPSK Waveform.
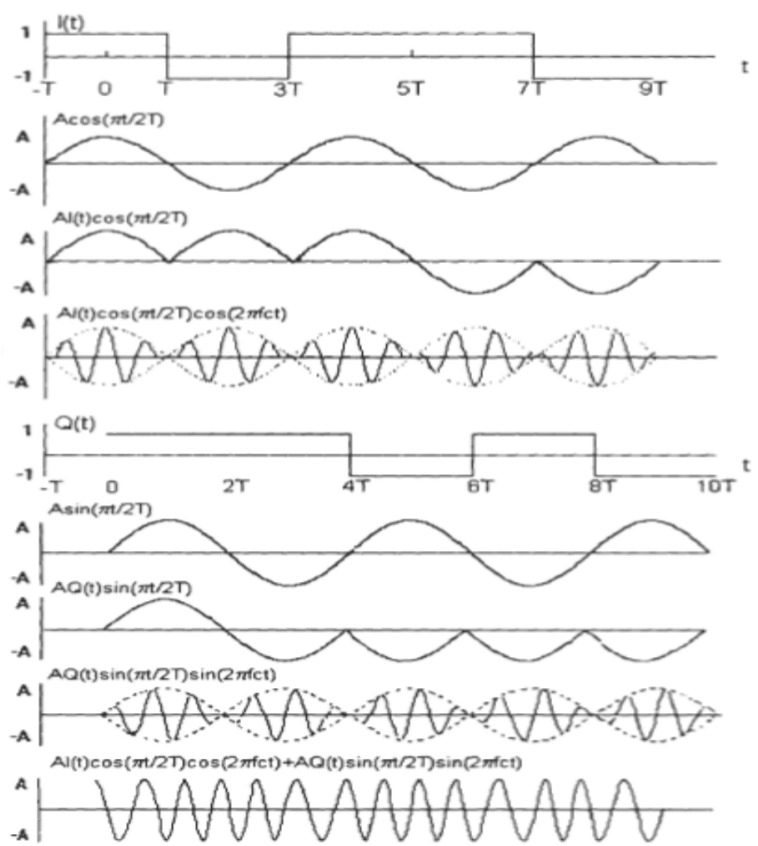

Fig. 7. MSK Modulation. 


\section{Proposed Model}

This paper focuses on implementing a Zigbee transmitter system using (PHY) layer specifications of IEEE 802.15.4 standard, and according to the features illustrated in Table 1.

Table 1,

Specifications of $2.4 \mathrm{GHZ}$ Zigbee Transmitter under IEEE 802.15.4 Standard

\begin{tabular}{ll}
\hline Features & Value \\
\hline Data Rate & $250 \mathrm{kbps}$ \\
Frequency of Operation & $2.4 \mathrm{GHZ}$ \\
Number of Channels & 16 \\
Channel Spacing & $5 \mathrm{MHZ}$ \\
Chip Rate & $2 \mathrm{Mbps}$ \\
Pulse Shaping & Half Sine \\
Spreading Technique & DSSS \\
Modulation Technique & OQPSK \\
\hline
\end{tabular}

The system is designed using MATLAB/Simulink, where the basic components of the proposed model are illustrated in Figure 8. The Following steps describe the design procedure in more details:

\section{Step1: Preparing of input bit stream}

This step includes applying mapping process of bit to symbol and symbol to chip. At first, the input data stream must be prepared to meet IEEE 802.15.4 specifications consider data rate and chip rate. In this design, random input generator from MATLAB/Simulink toolbox is used as a source of data stream, and it has been adjusted to operate on 250kbps.

Pseudo-random noise (PN) chip sequence is used to construct a direct sequence spread spectrum (DSSS). The reason behind the use of this technique is to increase the frequency of input data stream to 2 Mbps which in turn might improve transmitter performance in multi path environment. It is also used to achieve an improvement in signal to noise ratio (SNR) throughout increasing resistance of the system towards anticipated or accidental jamming 0 ][. 32 -bit chip sequence is generated at $2 \mathrm{Mbps}$ by $\mathrm{PN}$ sequence generator. Multiplying input data stream by a PN sequence after converting them into NRZ format produces DSSS output signal.

\section{step2: Serial to parallel conversion}

Prior to modulation process, the previous coded data are separated into In-phase and Quadrature data according to the scheme shown in figure 9. Even and odd clock pulses are generated by JK-flip flop in toggle state and with clock frequency equals to $2 \mathrm{MHZ}$. The positive output $\mathrm{Q}$ is used as a source of clock pulses for the first Dflip flop to produce the In-phase data with 1MHZ, while the negative output is fed to the second Dflip flop producing Quadrate data.

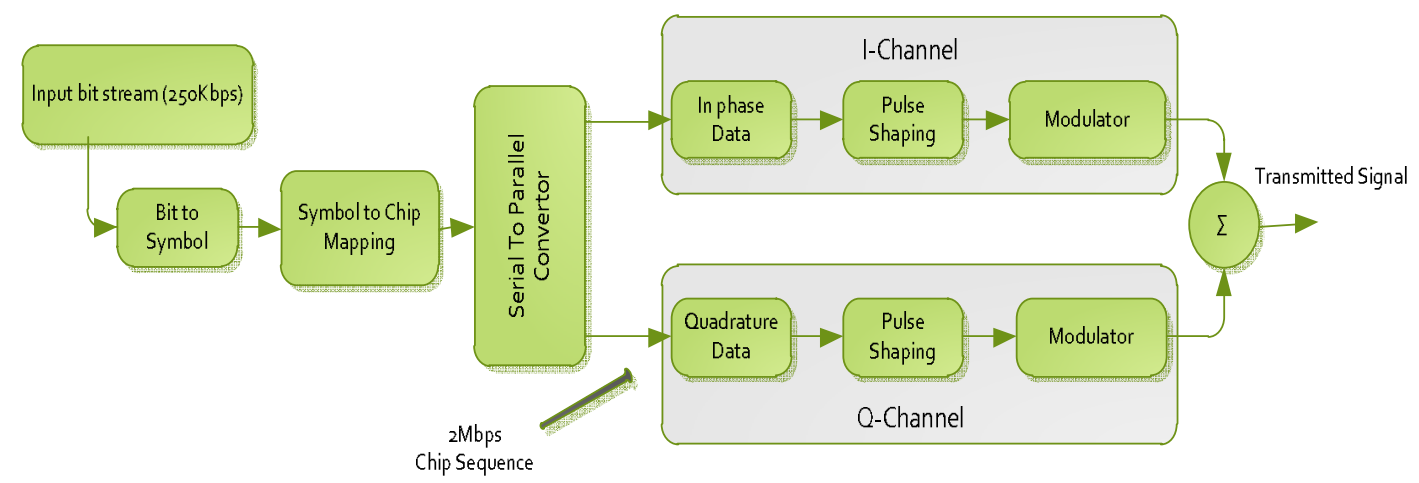

Fig. 8. Proposed Model.

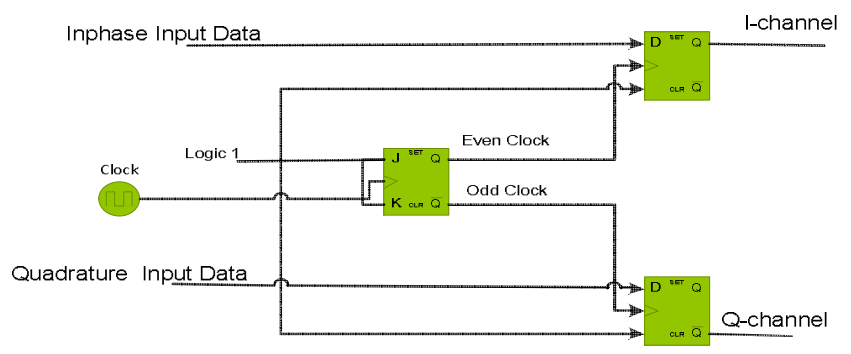

Fig. 9. Serial to Parallel Conversion 


\section{Step 3: Modulation Process}

OQPSK modulation is the same as QPSK except the Q-channel is delayed by T/2 seconds. Although the power density and error performance are the same in the two techniques, OQPSK produces a maximum phase change of $\pi / 2$ in contrast to QPSK which provides a phase shift of $\pi[12]$. The function of OQPSK signal for the modulator part can be written as [6]:

$$
s(t)=\frac{A}{\sqrt{2}} \mathrm{I}(\mathrm{t}) \cos 2 \pi f_{c} t-\frac{A}{\sqrt{2}} \mathrm{Q}\left(\mathrm{t}-\frac{\pi}{2}\right)
$$

$\sin 2 \pi f_{c} t,-\infty<t<\infty$

Where $A$ is the amplitude, $f_{c}$ is the center frequency, $\mathrm{I}(\mathrm{t})$ is the in-phase component, and $\mathrm{Q}\left(\mathrm{t}-\frac{\pi}{2}\right)$ is the shifted quadrature component of the signal. The output of In-phase and Quadrature data is firstly multiplied by half sine wave of $1 \mathrm{MHZ}$ to perform pulse shaping. The purpose behind the use of pulse shaping (also called premodulating filter) is to make the transmitted signal spectrum getting matched with channel bandwidth, hence; avoiding multipath and intersymbol interference (ISI) effects [5].In this design, half sine pulses are generated by multiplying sine wave having a frequency of 0.5 MHZ with train of pulses at $0.5 \mathrm{MHZ}$. In order to achieve synchronization state between In-phase and Quadrature data, a delay is added after the generated half sine pulses in I-Channel. The function of half sine pulse signal can be written as [11]:

$p(t)=\sin \frac{\pi t}{2 T_{c}}$

After half sine pulse shaping, the I-channel data are multiplied by a sine wave of $2.4 \mathrm{GHZ}$ and the Q-channel data is multiplied by a cosine wave at the same frequency. The final modulated transmitted signal is obtained by adding the output of I-channel and Q-channel. The simulated system is illustrated in figure 10 .

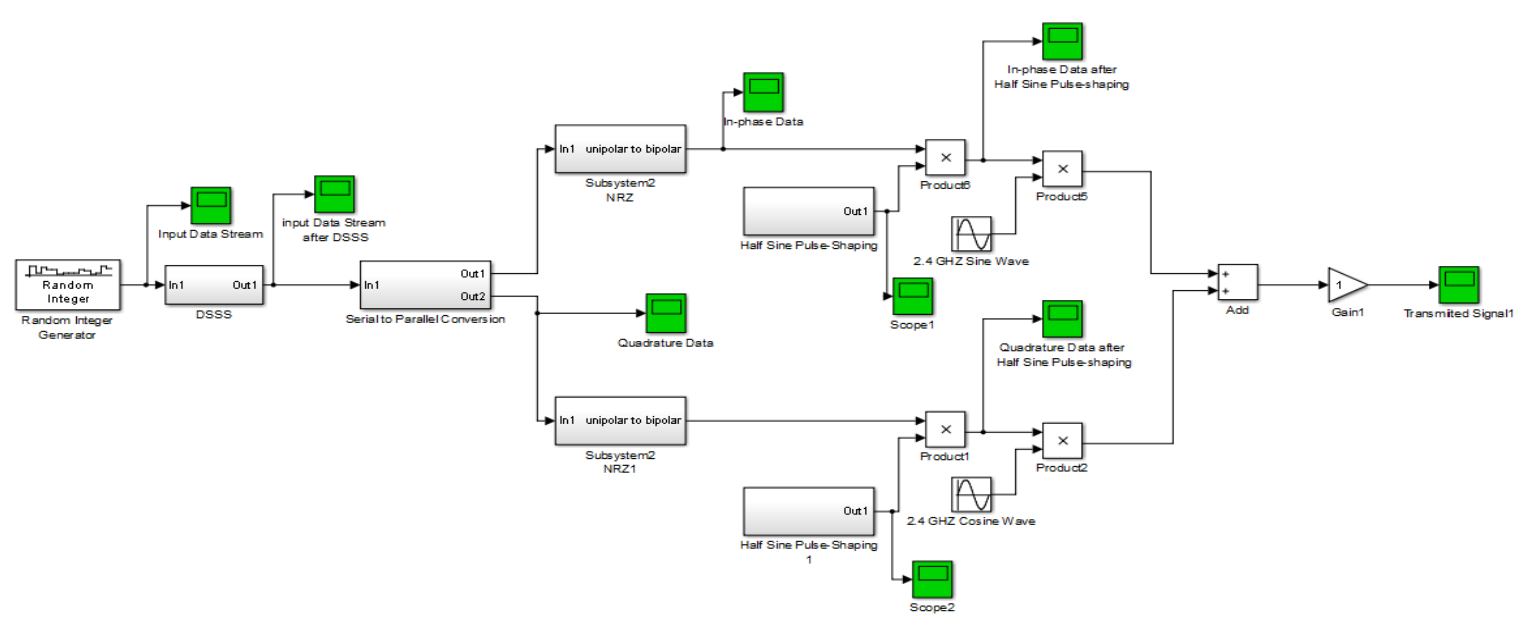

Fig. 10. Zigbee Transmitter with OQPSK Modulation and Half Sine Pulse-shaping.

\section{Simulation Results}

The proposed model has been implemented using MATLAB/Simulink to show system performance at each point of simulation where the following results have been gotten. The input bit stream has been produced by a random input generator with data rate of $250 \mathrm{kbps}$ as shown in figure 11 .

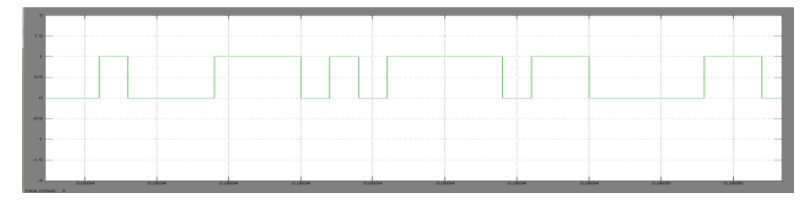

Fig. 11. Input Data Stream.

After generating input sequence, it has been multiplied by a PN sequence of 32 chips and chip rate of $2 \mathrm{Mbps}$ to produce a DSSS signal. Figures 12 and 13 depict the PN sequence and input data after applying spreading technique respectively. 
Fig. 12. The Output Signal PN Sequence Generator.

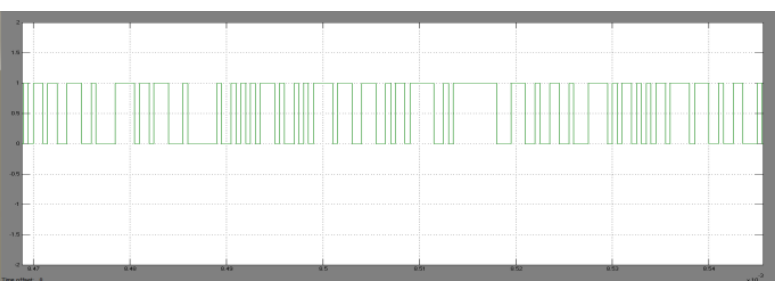

Fig. 13. Input data after Applying DSSS Technique.

The resulted signal is divided into even and odd part to produce the In-phase and Quadrature data by serial to parallel conversion and as shown in figure 14 and 15 respectively.

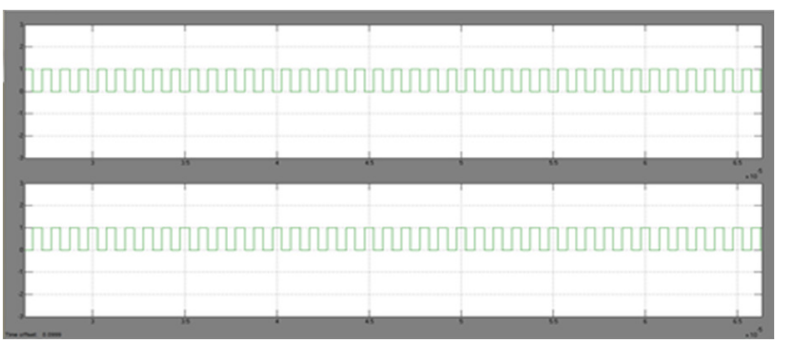

Fig. 14. Even and Odd Pulses.

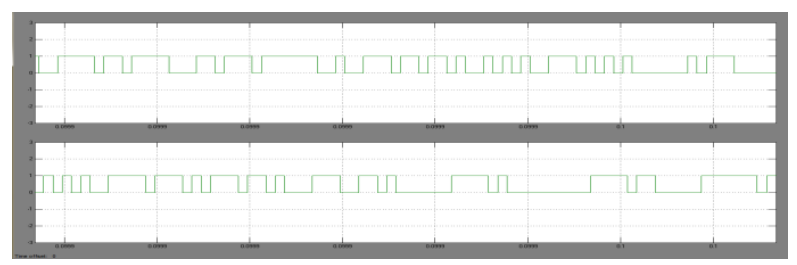

Fig. 15. In-phase and Quadrature Data.

The output data from In-phase and Quadrature channel are converted into NRZ format and then multiplied by a half sine wave. The figures below show the generation of half sine pulses and the output of each channel after employing this pulseshaping method.

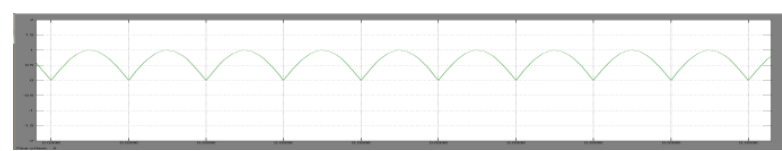

Fig. 16. Half Sine Pulse-shaping.

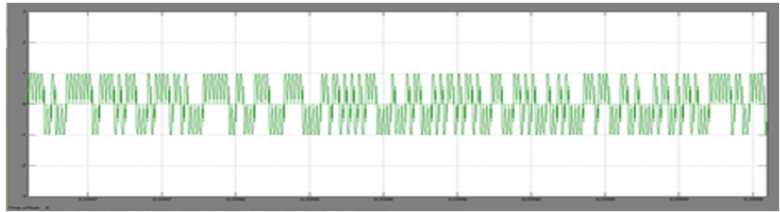

Fig. 17. In-phase Data after Half Sine PulseShaping.

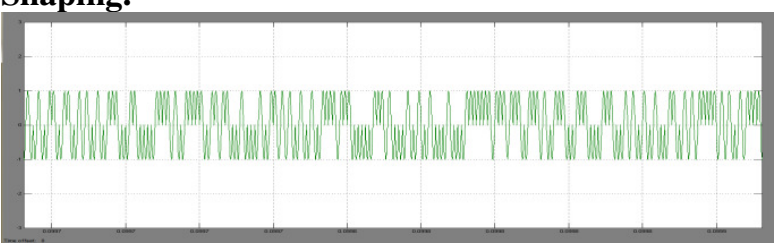

Fig. 18. Quadrature Data after Half Sine Pulse Shaping.

Figures 19 and 20 shows the output signal of In-phase and Quadrature channel after the modulation process; while the final output signal of Zigbee transmitter is depicted in figure 21.

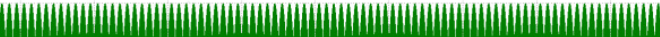

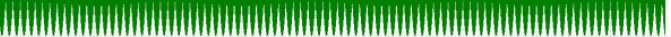

Fig. 19. In-phase Data after Modulation.

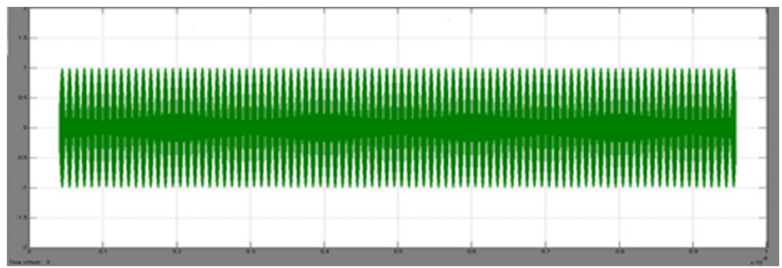

Fig. 20. Quadrature Data after Modulation.

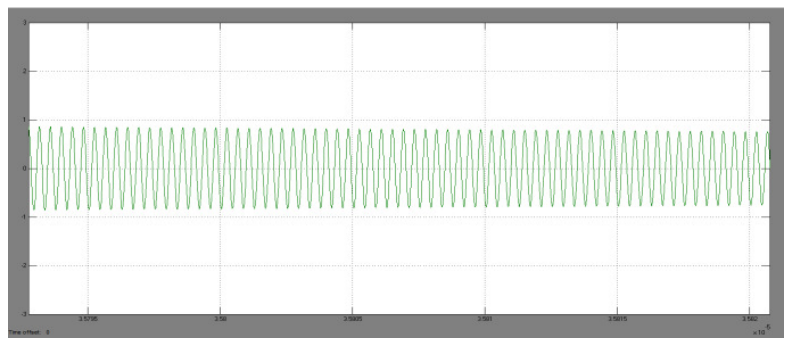

Fig. 21. Transmitted Signal of Proposed Zigbee Transmitter. 


\section{Conclusions}

In this paper, 2.4 GHZ Zigbee transmitter system is designed and simulated using MATLAB/Simulink to demonstrate the possible performance under IEEE 802.15.4specifications.

The system has been designed indirectly from scratch using OQPSK modulation technique with half sine pulse-shaping method. The result of simulation has shown that the waveform of the final signal was almost like minimum shift keying (MSK) modulator by assuring only a small amount of phase transitions (approximately less than $90^{\circ}$ ) because of the pulse-shaping.

Also, the use of direct sequence spread spectrum technique has the effect of further reducing the interference effects.

\section{References}

[1]S. Farahani, ZigBee wireless networks and transceivers: Newnes, 2011.

[2] N. A. Somani and Y. Patel, "Zigbee: A low power wireless technology for industrial applications," International Journal of Control Theory and Computer Modelling (IJCTCM), vol. 2, pp. 27-33, May 2012.

[3]I. M. El Emary and S. Ramakrishnan, Wireless Sensor Networks: From Theory to Applications: CRC Press, 2013.

[4]C. Wang, T. Jiang, and Q. Zhang, ZigBee® network protocols and applications: CRC Press, 2014.

[5] K. Gorantla and V. Mani, "Simulink model for Zigbee transceiver using OQPSK modulation under fading channels," IEEE International Conference in Communications and Signal Processing (ICCSP), pp. 0220-0224, 2015.

[6]F. Xiong, Digital modulation techniques: Artech House, 2006.

[7]D.K.Sharma, A.Mishra and Rajiv Savena "Analog \& Digital Modulation Techniques: An overview", International Journal of Computer Science and CommunicationTechnologies, Vol. No. 3, pp. 551-555, 2010.

[8]F. Quadri and A. D. Tete, "FPGA implementation of digital modulation techniques," IEEE International Conference in Communications and Signal Processing (ICCSP), Melmaruvathur, India, pp. 913-917, 2013.
[9] R. Ahmad, O. Sidek, W. M. H. W. Hassin, and S. K. K. Mohd, "Implementation of IEEE 802.15. 4-based OQPSKpulse-shaping block on FPGA," IEEE International Conference in Computer Applications and Industrial Electronics (ICCAIE), pp. 459-464, 2011.

[10] P. Mishra and S. Mane, "Implementation of QPSK Modulation on MATLAB Simulation," International Journal of Engineering and Innovative Technology (IJEIT), Volume 5, Issue 8, 2016

[11] R. Kanna, "Design of ZigBee transceiver for IEEE 802.15.4 using Matlab/Simulink," Master Thesis, Dept. Elec. Comm. Eng., National Institute of Technology, India, 2011.

[12] S. K. Kaul, "QPSK, OQPSK, CPM probability of error for AWGN and flat fading channels," Wireless Communication Technologies, 2005.

[13] W. Y. Yang, Y. S. Cho, W. G. Jeon, J. W. Lee, J. K. Kim, J. H. Paik, M. H. Lee, K. I. Lee, K. W. Park and K. S. Woo, "MATLAB/Simulink for digital communication", A-Jin Publishing, 2009.

[14] C. C. Wang, J. M. Huang, L. H. Lee, S. H. Wang, and C. P. Li, "A low-Power $2.45 \mathrm{GHz}$ ZigBee transceiver for wearable personal medical devices in WPAN," Digest of Technical Papers, International Conference on Consumer Electronics, pp. 1-2, 2007.

[15] M. B. Mantri, P. Velagapudi, B. C. Eravatri, and V. V. Mani "Performance Analysis of 2.4Ghz IEEE 802.15.4 PHY under various fading channels," IEEE International Conference in Emerging Trends in Communication, Control, Signal Processing Computing Applications (C2SPCA), pp.1-4, 2013.

[16] K. D. Jha and M. K. Srivastava, "Design \& simulation of zigbee transceiver system based on MSK and QPSK using MATLAB," International Journal of New Trends in Electronics and Communication (IJNTEC), Vol.4, Issue. 1, pp. 5-9, 2013. 


\section{تمثيل نظام إرسال Zigbee بالأعتماد على معيار IEEE 802.5.4 الخاص بشبكات المستشعر الألاكي مصنار

\author{
رسل هيثم هادية

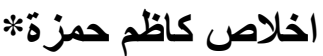

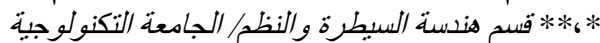

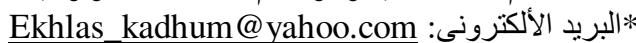 \\ russulh@gmail.com:البريد الأكترني:**
}

يعد Zigbee احد شبكات المستشعر اللاسلكي والذي صُمِمِ لملائمة التطبيقات ذات المدى القصير. يعنمد Zigbee في مو اصفات تصميمه على معيار

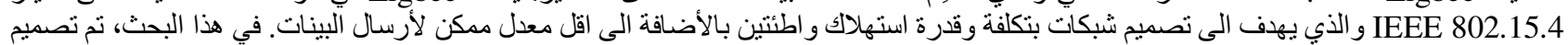

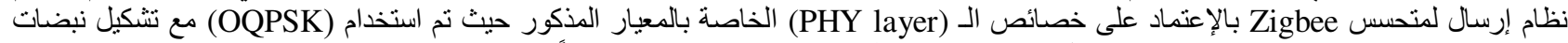

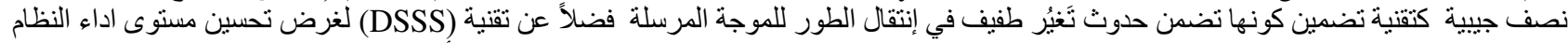
في البيئات متعددة القنو ات. تم تمثيل التصميم المقترح للنظام باستخدام برنامج MATLAB/Simulink لبيان الأداء بالنسبة لتقنية التضمين المطبقة. 